\title{
Non-zero-sum Dresher inspection games
}

\author{
Rudolf Avenhaus, Bernhard von Stengel \\ Informatik 5, Armed Forces University Munich \\ Werner-Heisenberg-Weg 39, D-8014 Neubiberg
}

Dedicated to the memory of Eckhard Höpfinger (1941-1990)

In: Operations Research '91, eds. P. Gritzmann et al., Physica-Verlag, Heidelberg 1992, 376-379.

An inspection game is here a non-cooperative two-person-game between an inspector and an inspectee. It models a situation where the inspector controls the inspectee who has an incentive to violate certain legal obligations. A recent survey of inspection games applied to data verification, for example in nuclear material safeguards, is given in [1].

Dresher [2] described a sequential inspection game of $n$ stages or time periods. The inspector can allocate $m$ inspections to these periods, $0 \leq m \leq n$. The inspectee knows at each stage the number of past inspections. He can decide to act legally or in at most one period to violate where he is caught iff the inspector simultaneously inspects. In [2], the game is zero-sum, with payoff zero for legal action and where the gain for the inspectee if he is undetected equals his loss if he is caught. This last assumption has been generalized by Höpfinger [3]. The present paper treats this game $\Gamma(n, m)$ with non-zero-sum payoffs. A closely related game with announced inspection strategies has been solved by Maschler [5].

\begin{tabular}{|c|c|c|}
\hline$I$ & legal action & \multicolumn{2}{|c|}{ violation } \\
\hline control & $V(n-1, m-1)$ & $-b$ \\
\hline no control & $I(n-1, m-1)$ & $-a$ \\
\hline
\end{tabular}

As in [2], the game $\Gamma(n, m)$ can be described recursively. For $0<m<n$, it is shown in the table as a two-by-two game with the choices of the inspector $I$ and the inspectee or "violator" $V$ at the first period. As will be shown by induction, the game has a unique equilibrium with payoffs $I(n, m)$ and $V(n, m)$ to $I$ and $V$. If $V$ violates at the first period, the game continues at the next stage like $\Gamma(n-1, m-1)$ respectively $\Gamma(n-1, m)$ depending on whether $I$ controls at the first stage or not. The equilibrium payoffs of these games are the entries in the first column of the table.

If $V$ violates at the first stage, he will get caught if $I$ controls and receive payoff $-b$ whereas $I$ gets $-a$. The absolute values of these payoffs are $a, b>0$. The negative sign 
emphasizes the undesirability of a caught violation for both players compared to legal action throughout the game which has reference payoff zero to both. For the inspector, even a caught violation usually represents a failure of the surveillance system that is supposed to induce legal behavior, so $I$ has a common interest with $V$ to have legal action instead, which cannot be modeled with a zero-sum game.

A violation at the first period without control does not terminate the game, but determines its payoffs, given by -1 to $I$ and 1 to $V$ (this and payoff zero for legal action normalizes the payoff functions which are unique only up to positive-linear transformations). The additional constraint $a<1$ indicates that $I$ prefers caught to uncaught violation. For $V$, the inequalities $1>0>-b$ show the reverse preference and an incentive to violate compared to legal action in order to model adequately the necessity of surveillance.

As pointed out by Kuhn [4, p.174], this recursive description of $\Gamma(n, m)$ tacitly assumes four subgames as a result of the actions of the players at stage one. This is actually not true if $I$ did not control since then he lacks information about what $V$ did, that is, in which subgame he is in. Nevertheless, $I$ can act as if no violation occurred since otherwise his actions are irrelevant because $V$ will then act legally and cannot be caught anymore. This can be justified formally by describing the game in extensive form with information sets. This description is crucial in a generalization to more than one admitted violation described in [6].

The equilibrium payoffs $I(n, m)$ and $V(n, m)$ of $\Gamma(n, m)$ are defined recursively, with the following base cases. If $m=0$, then $I$ has no inspections left and

$$
I(n, 0)=-1, \quad V(n, 0)=1 \quad \text { for } n>0
$$

since then $V$ can safely violate. If $m=n$, then $I$ can (and should) inspect at every remaining period so

$$
I(n, n)=0, \quad V(n, n)=0 \quad \text { for } n \geq 0
$$

since then $V$ prefers legal action; (2) applies also to $n=0$ where the game is over.

If $0<m<n$, the game table applies, which has a mixed equilibrium. Namely, assume as inductive hypothesis

$$
I(n-1, m-1)<I(n-1, m)
$$

that is, $I$ prefers not to control if $V$ acts legally (since $I$ can use the inspection later), which is true for $n=2, m=1$. If $V$ violates, $I$ prefers to control since $-a>-1$. Further inductive hypotheses are

$$
V(n-1, m-1)>-b \quad \text { and } \quad V(n-1, m)<1,
$$

also true for $n=2, m=1$, showing that $V$ prefers to act legally if controlled and illegally if not. These preferences are indicated by arrows at the sides of the table. $\Gamma(n, m)$ has 
then an equilibrium in mixed strategies: $I$ controls at the first period with the unique probability $p$ so that $V$ is indifferent between his actions,

$$
p \cdot V(n-1, m-1)+(1-p) \cdot V(n-1, m)=p \cdot(-b)+(1-p) \cdot 1 .
$$

Both terms of this equation denote the equilibrium payoff $V(n, m)$ to $V$ which after solving (5) for $p$ is

$$
V(n, m)=\frac{b \cdot V(n-1, m)+V(n-1, m-1)}{V(n-1, m-1)+b+1-V(n-1, m)} .
$$

Similarly, the probability $q$ for $V$ of legal action at stage one is determined by

$$
q \cdot I(n-1, m-1)+(1-q) \cdot(-a)=q \cdot I(n-1, m)+(1-q) \cdot(-1)
$$

yielding the equilibrium payoff $I(n, m)$ to $I$,

$$
I(n, m)=\frac{-a \cdot I(n-1, m)+I(n-1, m-1)}{I(n-1, m)-I(n-1, m-1)+1-a} .
$$

The functions $V(n, m)$ and $I(n, m)$ are uniquely defined by (1), (2), (6) and (7). Explicit formulas have been found by Dresher [2] for $b=1, a=-1$ and by Höpfinger [3] for the general zero-sum case $b>0, a=-b$ where $a<0$. This inequality $a<0$ can be weakened to $a<1$ as assumed here, with the solution from [3] given by

$$
I(n, m)=-\left(\begin{array}{c}
n-1 \\
m
\end{array}\right) / s(n, m) \quad \text { with } \quad s(n, m)=\sum_{i=0}^{m}\left(\begin{array}{c}
n \\
i
\end{array}\right)(-a)^{m-i} .
$$

This formula clearly fulfills (1) and (2), and (7) can be verified using the identities

$$
s(n-1, m)=-a \cdot s(n-1, m-1)+\left(\begin{array}{c}
n-1 \\
m
\end{array}\right)
$$

and

$$
s(n, m)=s(n-1, m)+s(n-1, m-1) .
$$

The explicit formula for $I(n, m)$ does not depend on the sign of the parameter $a$. With the formal substitutions $V(n, m)=-I(n, m)$ and $b=-a$, the base cases $(1),(2)$ and the recurrences (6) and (7) are equivalent, so

$$
V(n, m)=\left(\begin{array}{c}
n-1 \\
m
\end{array}\right) / t(n, m) \quad \text { with } \quad t(n, m)=\sum_{i=0}^{m}\left(\begin{array}{l}
n \\
i
\end{array}\right) b^{m-i} .
$$

To complete the induction, the inequalities (4) are seen immediately and (3) is seen as follows: the term $s(n, m)$ is positive since it can also be defined recursively by $s(n, 0)=1$, $s(n, n)=(1-a)^{n}$ (both positive since $\left.a<1\right)$ and by $(9)$ for $0<m<n$. Similarly, one obtains $s(n-1, m)>\frac{n-1-m}{m} \cdot\left(\begin{array}{c}n-1 \\ m-1\end{array}\right)$ for $0<m<n$. Since $s(n-1, m-1) \leq\left(\begin{array}{c}n-1 \\ m-1\end{array}\right)$ by (8) with $m-1$ instead of $m$, this implies $s(n-1, m)>\frac{n-1-m}{m} \cdot s(n-1, m-1)$ or $(3)$. 
The probability $p$ of control at the first period in (5) can be simplified to the expression $p=t(n-1, m-1) / t(n, m)$. (A similarly simple expression for $q$ is not known.) This describes the inspector strategy for each stage, which depends on the number of inspections used so far. The resulting unconditional probabilities of control are equal for the first $n-m$ periods and monotonically increasing afterwards, where this increase is the higher the greater $b$ is. For $m=1$, for example, control in one of the first $n-1$ periods is equally probable and in the last period $1+b$ times as probable. This meets the intuition that sequential inspections should in tendency be accumulated towards the end to be optimal.

Finally, the solution of the game $\Gamma(n, m)$ holds also if the constraints $1>a>0$ and $b>0$ are weakened, since the recurrences (6) and (7) are valid as long as the payoff table has the circular structure indicated by the arrows. Keeping the basic equations (1) and (2), the number $a$ can be any real less than 1 , where for $a \leq 0$, the inspector receives a nonnegative "reward" $-a$ (then better denoted by an unsigned variable) when he catches the inspectee. This includes the zero-sum game with $-a=b$ that, by induction, results into $V(n, m)=-I(n, m)$. The value $I(n, m)$ of this game has the recurrence $(7)$, which allows to use Höpfinger's [3] solution of this case as mentioned above.

Formally, the solution of $\Gamma(n, m)$ is also valid if the constraint $b>0$ is weakened to $b>-1$, but for $b<0$ the inspectee would even with a caught violation gain over legal behavior, which invalidates the assumption $V(n, n)=0$ in (2). In the case $b=0$, admitted in [6] for generalizations of $\Gamma(n, m)$, the inspectee neither gains nor loses with a caught violation. It can be seen that then the optimal inspection scheme is very uniform through all stages, which has interesting applications cited in [6, section 5].

\section{REFERENCES}

[1] Avenhaus, R. and B. von Stengel, Current research in data verification. Contribution to the IMACS International Workshop on Decision Support Systems and Qualitative Reasoning, Toulouse, France, March 13-15 (1991)

[2] Dresher, M., A sampling inspection problem in arms control agreements: a gametheoretic analysis. Memorandum No. RM-2972-ARPA, The RAND Corporation, Santa Monica, California (1962)

[3] Höpfinger, E., A game-theoretic analysis of an inspection problem. C-Notiz No. 53, University of Karlsruhe, preprint (1971)

[4] Kuhn, H.W., Recursive inspection games. In: Applications of Statistical Methodology to Arms Control and Disarmament, eds. F.J. Anscombe et al., Final report to the U.S. Arms Control and Disarmament Agency under contract No. ACDA/ST-3 by Mathematica, Inc., Princeton, New Jersey (1963), Part III, pp. 169-181

[5] Maschler, M., A price leadership method for solving the inspector's non-constant-sum game. Naval Research Logistics Quarterly 13 (1966), 11-33

[6] von Stengel, B., Recursive inspection games. IASFOR-Bericht S-9106, Fakultät für Informatik, Armed Forces University Munich (1991) 